\title{
Sprouty proteins, masterminds of receptor tyrosine kinase signaling
}

\author{
Miguel A. Cabrita · Gerhard Christofori
}

Received: 14 December 2007 / Accepted: 7 January 2008/Published online: 25 January 2008

(C) Springer Science+Business Media B.V. 2008

\begin{abstract}
Angiogenesis relies on endothelial cells properly processing signals from growth factors provided in both an autocrine and a paracrine manner. These mitogens bind to their cognate receptor tyrosine kinases (RTKs) on the cell surface, thereby activating a myriad of complex intracellular signaling pathways whose outputs include cell growth, migration, and morphogenesis. Understanding how these cascades are precisely controlled will provide insight into physiological and pathological angiogenesis. The Sprouty (Spry) family of proteins is a highly conserved group of negative feedback loop modulators of growth factor-mediated mitogen-activated protein kinase (MAPK) activation originally described in Drosophila. There are four mammalian orthologs (Spry1-4) whose modulation of RTK-induced signaling pathways is growth factor - and cell context - dependant. Endothelial cells are a group of highly differentiated cell types necessary for defining the mammalian vasculature. These cells respond to a plethora of growth factors and express all four Spry isoforms, thus highlighting the complexity that is required to form and maintain vessels in mammals. This review describes Spry functions in the context of endothelial biology and angiogenesis, and provides an update on Spry-interacting proteins and Spry mechanisms of action.
\end{abstract}

Keywords EGF - Endothelial cell - ERK - FGF . Growth factors - MAPK - Receptor tyrosine kinase . Sprouty $\cdot$ Spred $\cdot$ VEGF

M. A. Cabrita · G. Christofori ( $\square)$

Institute of Biochemistry and Genetics, Department of

Biomedicine, University of Basel, Mattenstrasse 28, 4058 Basel,

Switzerland

e-mail: gerhard.christofori@unibas.ch

\begin{tabular}{|c|c|}
\hline \multicolumn{2}{|c|}{ Abbreviations } \\
\hline Ang & Angiopoietin \\
\hline $\mathrm{c}-\mathrm{Cbl}$ & $\begin{array}{l}\text { Cellular homologue of Casitas B-lineage } \\
\text { lymphoma proto-oncogene product }\end{array}$ \\
\hline EGF & Epidermal growth factor \\
\hline EGFR & EGF receptor \\
\hline eNOS & Endothelial nitric oxide synthase \\
\hline ERK & Extracellular signal-regulated kinase \\
\hline FGF & Fibroblast growth factor \\
\hline FGFR & FGF receptor \\
\hline GDNF & Glial-derived neurotrophic factor \\
\hline Grb2 & Growth factor receptor-bound protein 2 \\
\hline HMVEC & Human microvascular endothelial cell \\
\hline Hrs & $\begin{array}{l}\text { Hepatocyte growth factor-regulated tyrosine } \\
\text { kinase substrate }\end{array}$ \\
\hline HUVEC & Human umbilical vein endothelial cell \\
\hline MAPK & Mitogen-activated protein kinase \\
\hline MEK & MAPK and ERK kinase \\
\hline Mnk1 & $\begin{array}{l}\text { Mitogen-activated protein kinase-interacting } \\
\text { kinase } 1\end{array}$ \\
\hline PDGF & Platelet-derived growth factor \\
\hline PKC & Protein kinase $\mathrm{C}$ \\
\hline PLC & Phospholipase C \\
\hline PP2A & Protein phosphatase $2 \mathrm{~A}$ \\
\hline RBD & Raf1-binding domain \\
\hline RTK & Receptor tyrosine kinase \\
\hline Shp2 & $\begin{array}{l}\text { SH2-domain-containing protein } \\
\text { phosphatase } 2\end{array}$ \\
\hline SIAH2 & Seven-in-Absentia Homolog 2 \\
\hline SMC & Smooth muscle cell \\
\hline Sos1 & Son of Sevenless 1 \\
\hline Spry & Sprouty \\
\hline Spred & $\begin{array}{l}\text { Spry-related proteins with Enabled/vasodilator- } \\
\text { stimulated phosphoprotein homology } 1 \text { domain }\end{array}$ \\
\hline SPR & Spry-related domain \\
\hline
\end{tabular}


VEGF Vascular endothelial growth factor

VEGFR VEGF receptor

\section{Introduction}

Receptor tyrosine kinases (RTKs) have been at the forefront of cancer research for many years due to their pivotal role in cancer biology as prognostic indicators and therapeutic targets $[1,2]$. Thus, the elucidation of RTK signaling cascades and their regulatory networks has been an important objective of many laboratories, especially since angiogenesis and the angiogenic switch necessitate activation of various growth factor receptors [3-5]. The formation of the vasculature by the endothelium and supporting stroma via branching morphogenesis is analogous to what is observed in the development of tubular networks found in other tissues

a

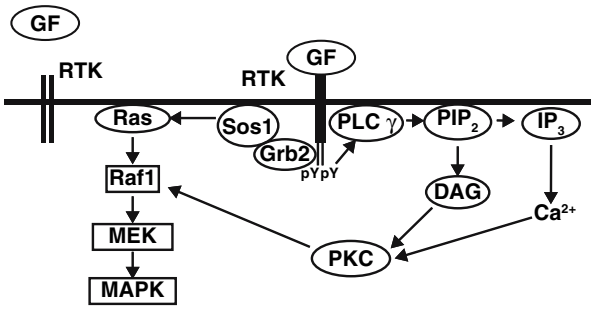

C

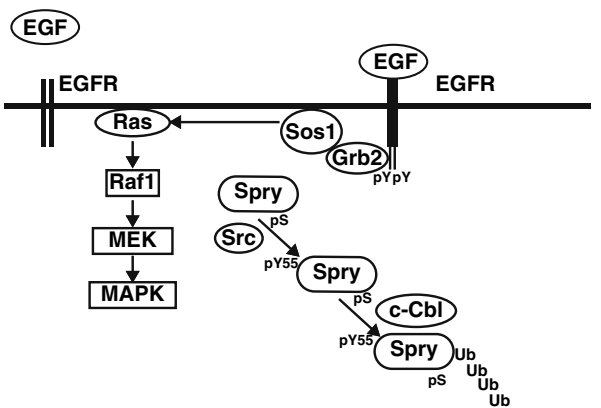

Fig. 1 Spry mechanisms of action in growth factor signaling pathways. (a) Canonical signal transduction in the absence of Spry. Upon ligand binding, RTKs can activate MAPK in a Ras-dependent manner via the adapter protein Grb2 and Sos1-mediated activation of Ras, Raf1, MEK, and eventually MAPK. Another conserved RTK pathway that can be activated is that mediated by PLC $\gamma$, which converts phosphatidylinositol $[4,5]$-bisphosphate $\left(\mathrm{PIP}_{2}\right)$ into two second messengers: inositol [1, 4, 5] trisphosphate $\left(\mathrm{IP}_{3}\right)$ and diacylglycerol (DAG), leading to calcium $\left(\mathrm{Ca}^{2+}\right)$ mobilization. Both DAG and $\mathrm{Ca}^{2+}$ activate PKC, which in turn phosphorylates Raf1, thus leading to MAPK activation. (b) Spry-mediated inhibition of the FGF signaling pathway. Spry is serine phosphorylated on multiple residues in unstimulated cells and at least serines 112 and 115 are dephosphorylated by PP2A upon FGF stimulation [23, 57]. Also, Spry phosphorylation at Y55 by a Src-like kinase and by an unknown kinase (X) at Y227 is required for Spry to inhibit FGF-induced activation of MAPK [24, 48, 52, 56]. Under some conditions, Spry (e.g. lung, kidney, mammary gland, and placenta) $[6,7]$. Growth factors, such as the vascular endothelial growth factors (VEGFs), fibroblast growth factors (FGFs), epidermal growth factor (EGF), ephrins, and angiopoietins (Angs), in conjunction with their cognate receptors play a crucial role in blood vessel formation in normal and pathological settings (reviewed in $[8,9]$ ). The majority of RTKs, serving as gatekeepers for these extracellular growth factors, among several signaling pathways, activate the extracellular signalregulated kinase (ERK)/mitogen-activated protein kinase (MAPK) signaling cascade composed of Raf1, MAPK and ERK kinase (MEK), and MAPK (Fig. 1a). These pathways are precisely controlled spatio-temporally by the activity of various modulatory proteins in a cell context-dependant manner [10-12].

In vertebrates, such modulators of RTK signaling include the members of the Sprouty (Spry) family of proteins, comprising various Spry and Spred (Spry-related proteins with b

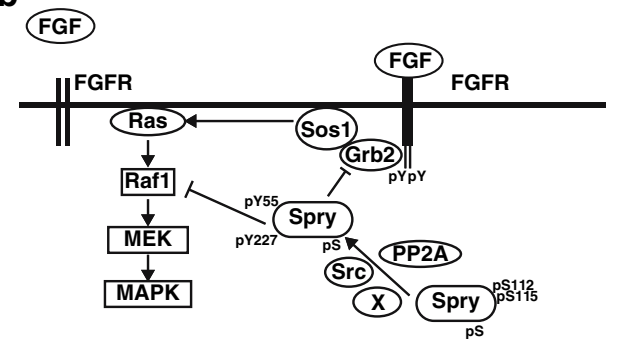

d

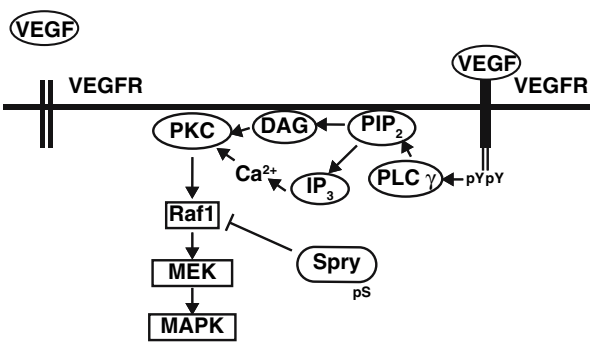

binding to Grb2 is sufficient to inhibit FGF-induced activation of MAPK, while in others Grb2 association is not required [42, 55]. Also, Spry binding to Sos1 and potentially to Raf1 inhibits FGFstimulated MAPK activity [18, 43]. (c) Spry-mediated potentiation of the EGF signaling pathway. Upon EGF binding, Spry is tyrosine phosphorylated and competes with EGFR for binding to c-Cbl. By interacting with c-Cbl, Spry becomes ubiquitinylated and eventually degraded, allowing for sustained EGFR signaling [44-46, 61, 80]. (d) Spry-mediated inhibition of the VEGF signaling pathway. The VEGFR signals via the PLC $\gamma /$ PKC pathway [66]. Upon VEGF activation, Spry interacts with Raf1 via its RBD and inhibits activation of MAPK. Tyrosine phosphorylation of Spry is not required for this activity [67]. pY and $\mathrm{pS}$ denote phosphorylated tyrosine and serine residues, respectively. In this schematic, using Spry2 as the representative Spry isoform, pY55 and pY227 represent the $\mathrm{N}$ - and $\mathrm{C}$ - terminal phospho-tyrosines 
Enabled/vasodilator-stimulated phosphoprotein homology 1 domain) isoforms. Depending on the cell type and physiological conditions, Sprys and Spreds modulate RTK signaling (and sometimes also signaling by G-protein-coupled receptors) by mainly repressing the MAPK pathway (see below). The founding member of the family, Drosophila Spry (dSpry), was initially described as a negative feedback loop inhibitor of FGF-mediated branching of the Drosophila trachea [13]. There are four mammalian dSpry homologs, Spry1-4 (reviewed in [14-18]) and four Spred proteins, Spred1-3 and EVE-3 (a splice variant of Spred-3) (reviewed in [19]) that all contain the highly conserved cysteine-rich Sprouty-related (SPR) domain. The expression of Spry1, 2, and 4 is widespread in embryos and adults, while that of Spry3 is believed to be more restricted (brain and testes in adult tissues) [20, 21]. Like dSpry, the expression of mammalian Spry proteins is induced by the growth factor cascades that they target [22-24]. Upon growth factor activation, a subset of Spry proteins translocate to the cell membrane where MAPK inhibition presumably occurs [23, $25,26]$. Spry proteins can modulate MAPK activation induced by several stimuli, including EGF, FGF, VEGF, platelet-derived growth factor (PDGF), nerve growth factor, hepatocyte growth factor, insulin, stem cell factor/kit ligand, glial-derived growth factor (GDNF), brain-derived neurotrophic factor, and T-cell receptor activation [17, 18, 22, 24, 27-30]. This review will discuss Spry protein function in the context of endothelial cells and provide an update on their mechanism of action, Spry-interacting partners, and regulation of Spry stability and activity.

\section{Spry expression}

Endothelial cells can be cultured in vitro to form capillarylike structures and various molecular approaches have been employed to identify genes required for tube/capillary formation. For example, using subtractive cDNA library hybridization, proliferating human microvascular endothelial cells (HMVECs) were compared to those that differentiate and form tube-like structures on Matrigel [31]. These studies identified several transcripts, including Spry2 mRNA, which were differentially enriched in tube-forming HMVECs versus proliferating HMVECs. Also, in an early DNA microarray study, Spry 1 was found highly upregulated in human umbilical vein endothelial cells (HUVECs) during capillary morphogenesis in a three-dimensional collagen matrix [32]. The increase in Spry 1 mRNA abundance coincided with a decrease in FGF2 mRNA. Another mRNA following a pattern similar to that of Spry1 was Ang2, an antagonistic ligand of the Tie 2 receptor, and one of the most upregulated transcripts detected in this experiment [33]. Similarly, Spry1, along with Ang2 and other mRNAs, was found enriched in microvascular endothelial cells, suggesting a specific role for Spry1 in capillary tube formation [34]. More recently, a comprehensive expression analysis of FGFs, FGF receptors (FGFRs), and various FGF signaling inhibitors was performed in primary human endothelial and vascular smooth muscle cells (SMCs) [35]. This study demonstrated that HUVECs and SMCs express a variety of FGFs (FGF1, 2, 5, 7, 8, 11, 12, 16, 18), FGFRs (FGFR1c, 2c, $3 \mathrm{c})$ and FGF signaling antagonists including all four Spry isoforms. Furthermore, in spheroid co-cultures consisting of HUVECs and SMCs, the repertoire of these various components of the FGF signaling cascade did not significantly change, suggesting that both cell types behaved as an entity rather than as two distinct cell populations. Thus, endothelial cells express all four isoforms of Spry, underlining the need for specialization, yet maintaining redundancy in these highly differentiated cells.

More recently, additional insights into the regulation of Spry expression in endothelial cells have been reported [36]. The highly conserved FoxO genes, FoxO1, FoxO3a, and FoxO4, are expressed in endothelial cells, and null mice for FoxO1 and FoxO3 display vascular phenotypes (reviewed in [37]). Moreover, mice deficient for all three FoxO genes exhibited tissue-specific hemangiomas resulting in premature death [36]. Spry2 was identified by microarray analysis as an important FoxO target gene in affected endothelial cells. The Spry 2 promoter contained FoxO-binding elements, and Spry2 expression correlated with FoxO deletion in a tissue-specific manner. Furthermore, in liver endothelial cells, Spry expression was lost upon FoxO deletion, an effect not observed in lung endothelial cells. Phenocopying Spry2 loss by RNA interference technology confirmed that endothelial cells lacking Spry2 were more proliferative and less susceptible to apoptosis. Apparently, both the Fox and the Ets transcription factor families are required for proper angiogenesis, and for endothelial cell function and differentiation [37]. Also, the human Spry2 gene promoter has been shown to harbor a functional binding element for Ets-1, suggesting that it is a target of the Ets transcription factor family [38].

\section{Spry functions}

In initial experiments to characterize the function of Spry 1 and Spry2, HUVECs were employed to examine their role in VEGF, FGF, and EGF signaling [23]. Forced expression of Spry1 and Spry2 in endothelial cells inhibited VEGF-, FGF-, and EGF-stimulated growth and sprouting. Interestingly, these Spry isoforms only repressed FGF- and VEGFinduced phosphorylation of MAPK and not that stimulated by EGF, demonstrating that various RTK cascades were differentially affected by Spry proteins. In another study, 
Spry4 function was examined in endothelial cells using a combination of in vitro and in vivo experiments [39]. Endothelial cell proliferation, migration, and MAPK activation induced by either FGF2 or VEGF were inhibited by Spry4 expression, and the Spry4-expressing cells arrested in the $G_{1} / G_{2}$ phase of the cell cycle. Notably, Spry4 inhibition could be overcome by a constitutively active form of Ras, suggesting that Spry4 exerted its functions upstream of Ras. Moreover, the cell cycle inhibitor p21 was induced and phosphorylation of the cell cycle control protein $\mathrm{Rb}$ was decreased, which correlated with the presence of Spry4 in these cells. Lastly, gene transfer-mediated expression of Spry4 in the vascular endothelium of the mouse embryo resulted in marked repression of sprouting and branch formation of the yolk sac vasculature [39]. Thus, Spry proteins are important regulators of RTK function (i.e. growth and differentiation) in endothelial cells and inhibit angiogenesis in in vitro and in vivo model systems.

\section{Spry mechanisms of action}

The exact mode of action for Spry proteins has been difficult to ascertain, as noted by early reports examining the functional role of the various Spry isoforms in different growth factor pathways in diverse cell types and species [15-18, 40]. Vertebrate Spry proteins are now viewed as general modulators of growth factor signaling, and for most RTK signaling cascades, Spry proteins repress MAPK activation. For FGF signaling, all mammalian Spry proteins inhibit FGF-induced MAPK activation to varying degrees [41-43]. However, in the case of EGF stimulation, Spry2 can potentiate MAPK activation in some cell types [44-46]. This context-specific dual function of Spry has also been illustrated for T-cell receptor signaling where Spry1 acts either as an inhibitor or as an enhancer depending on the differentiation state of the T-cell [30]. In short, Spry modulation of RTK signaling is very pliable and highly cell- and context-dependent.

All Spry isoforms possess a highly conserved N-terminal tyrosine residue, which in the case of Spry1 and Spry2 is phosphorylated upon EGF and FGF signaling and in the case of Spry4 is phosphorylated upon FGF and insulin signaling [24, 42, 47, 48]. Mutation of this tyrosine generates a dominant-negative Spry unable to inhibit FGFstimulated MAPK activation [24, 48, 49]. The Src family of kinases is likely responsible for phosphorylation of Spry at this conserved tyrosine, while Shp2 phosphatase is involved in dephosphorylating this critical residue [48, 50-52].

\section{FGF signaling}

The FGF/FGFR signaling axis is important for endothelial growth, migration, and morphogenesis and is critical for angiogenesis in both normal and disease states [53]. To elucidate the Spry mechanism of action, most studies have focused on Spry2, the most highly conserved isoform across vertebrates. Initially, both Spry1 and Spry2 have been proposed to bind Grb2 via the N-terminal conserved tyrosine residue, thus interrupting the signaling cascade before MAPK can be activated [22, 43, 45, 49, 54] (Fig. 1b). The role of Grb2 in Spry-mediated inhibition of FGF-induced MAPK pathway has remained controversial. Lao and co-workers discovered, using an FGFR overexpression system (i.e. long-term MAPK signaling), that Spry2 requires an FGFR-induced conformational change involving a cryptic PXXPXR motif on its C-terminus to bind Grb2 via its N-terminal SH3 domain [42]. However, a study involving acute FGF signaling (i.e. short-term MAPK activation without FGFR overexpression) demonstrated an uncoupling of Spry2-Grb2 binding from Sprymediated inhibition of MAPK activation [55]. This work revealed that Spry2 harbors two Grb2-binding sites and that a mutant hSpry2 lacking both sites still antagonized FGF signaling. Thus, how FGF signaling is antagonized by Spry may depend on the type and/or duration of the original stimulus.

Post-translational modification of Spry likely plays a central role in determining its function and activation status. In unstimulated cells, Spry1 and Spry2 have been found phosphorylated on serine residues [23]. Spry2 inhibition of FGF signaling requires phosphorylation of tyrosine 227 (Y227), which is dispensable for Spry2 potentiation of EGF signaling [56]. Recently, Lao and coworkers reported that a change in Spry2 phosphorylation status was required for the inhibition of FGFR-induced MAPK activation [57]. Upon FGFR activation, Spry2 is dephosphorylated by the protein phosphatase 2A (PP2A) at highly conserved serine residues (S112 and S115) in the Spry N-terminus, thus activating Spry [57]. PP2A competes with the E3 ubiquitin ligase, c-Cbl, for binding to Spry2, thus establishing two pools of Spry2-containing complexes and striking a balance between Spry2 activation and degradation. Hence, both tyrosine phosphorylation and serine dephosphorylation are required for Spry2-mediated inhibition of FGFR signaling (Fig. 1b).

Another characteristic of Spry proteins is their ability to form homo- and hetero-complexes [24, 41, 48, 49]. In the context of FGF signaling all four Spry isoforms have been reported to interact with each other via their cysteine-rich domains [43]. In a heterodimer of Spry1 and Spry4, Spry1 can bind to Grb2, while Spry4 interacts with Sos1. These studies also revealed that the interaction between Spry1 and Spry4 was the most potent combination for inhibiting FGF2-induced MAPK activation. Thus, the combinatorial set of Spry isoforms within a cell determines the extent of FGF signaling inhibition. 


\section{EGF signaling}

The role and components of the EGF-induced MAPK cascade in normal endothelium are still under debate; however, there is consensus that EGF signaling plays an important role in tumor endothelium [58-60]. The function of Spry in the EGF signaling cascade has been the most controversial due to the different cell types that have been examined. The general consensus is that Spry can potentiate EGF-induced MAPK activation in certain cellular contexts. The EGF receptor (EGFR) is normally rapidly downregulated due to its interaction with the E3 ubiquitin ligase, c-Cbl. However, Spry2 interferes with this interaction and competes with the EGFR for c-Cbl-binding preventing receptor internalization, thus sustaining EGFRmediated signaling [44, 46, 61, 62] (Fig. 1c). Moreover, Spry2 binds CIN85, an endocytic adapter, thus regulating the clustering of $\mathrm{c}-\mathrm{Cbl}$ that is required for EGFR endocytosis and degradation [63]. Other RTKs, such as the FGFR, are also degraded by c-Cbl, and it is still not clear how Spry discriminates between EGF and FGF signaling. In a recent study, where Spry2 levels were reduced by RNA interference, FGF signaling was increased, while EGF signaling was reduced [56]. These experiments led to the discovery that phosphorylation at a conserved tyrosine (Y227) discriminates between the EGF and FGF signaling pathways, suggesting that unidentified proteins bind to this C-terminal region depending on the signaling context. More recently, Kim et al. [64] described that Spry2 interfered with activated EGFR trafficking to late endosomes. Spry2 interacts with hepatocyte growth factor-regulated tyrosine kinase substrate (Hrs) in early endosomes. The Spry2-Hrs complex disrupts the Hrs association with tumor susceptibility gene 101, an interaction required for EGFR movement from early to late endosomes, resulting in delayed EGFR trafficking and perturbation of phospho-MAPK accumulation in late endosomes. Thus, potentiation of EGF signaling by Spry relies on interactions with proteins that are involved in the endocytosis and degradation of EGFR.

\section{VEGF signaling}

VEGF-induced signal transduction is the most critical and best studied signaling pathway in endothelial cells and plays a central role in vascular development and in physiological and pathological angiogenesis [65]. In endothelial cells, VEGF binds to VEGF receptor (VEGFR) and activates MAPK via the phospholipase C gamma (PLC $\gamma$ ) and protein kinase $\mathrm{C}$ (PKC) pathway where PKC phosphorylates Raf1, thereby activating ERK/MAPK in a Rasindependent manner [66] (Fig. 1a). Here, Spry-mediated inhibition of VEGF signaling occurs via a different mechanism as compared to FGF and EGF signaling [67] (Fig. 1d). Spry4 binds to Raf1 via a highly conserved Cterminal domain, thus ablating VEGF signaling by segregating Raf1 away from the cascade. All Spry isoforms carry this Raf1-binding domain (RBD) in their highly conserved C-terminal domain suggesting that all Spry isoforms may be capable of inhibiting VEGF signaling in a similar manner.

Apparently, Spry mechanisms of action are as varied as their target RTKs. For each signaling cascade studied in depth so far, Spry proteins have evolved an inhibitory strategy unique to each pathway.

\section{Spry-interacting proteins and Spry activity}

Spry proteins have accumulated a long list of interacting and associated proteins that regulate Spry function, stability, and localization, many of which interact with the Cterminal SPR domain (reviewed in [17, 18]). Included in this list is Caveolin-1, a transmembrane protein and the major architectural component of caveolae, flask-shaped pits on the cell membrane [68]. Caveolin-1 promotes capillary tube formation, and its role in regulating angiogenesis has been underlined in transgenic overexpression and knockout experiments in mice [69-72]. Caveolin-1 also inhibits RTK signaling, yet in a cell confluencydependent manner [73]. Caveolin-1 co-localizes and immunoprecipitates with Spry1 and Spry2 [23]. Upon further analysis, all Spry isoforms interact with Caveolin-1 and Spry binds to Caveolin-1 via its highly conserved cysteine-rich C-terminal domain [41]. Caveolin-1 in turn binds to the various Spry isoforms via two domains, a Nterminal and a C-terminal domain. Spry proteins do not require Caveolin-1 for their function nor for their membrane targeting; however, Caveolin-1 acts cooperatively with some Spry isoforms depending on whether the EGF or FGF pathways are being stimulated. In summary, Caveolin-1 modulates Spry activity in an isoform-, cell density-, and growth factor-specific manner, suggesting that together these two proteins fine tune growth factor signaling.

Recently, Necl-5, also known as poliovirus receptor/ CD155/Tage4, has been identified as a novel Spry2-interacting protein [74]. Necl-5 is a mediator of contact inhibition that forms a complex with integrin $\alpha_{\mathrm{v}} \beta_{3}$, is upregulated by PDGF and FGF, and is also found on endothelial cells [74-76]. Necl-5 interacts with Spry2 in a cell confluency-dependent manner and controls Spry2 tyrosine phosphorylation, thus modulating the PDGF-activated MAPK signaling pathway. This novel binding partner demonstrates once again the importance of cell-cell contacts in the fine-tuning of Spry-mediated inhibition of RTKs. 
Another previously described Spry-interaction partner, TESK1, a cofilin kinase, has now been shown to interact with all Spry proteins [77]. Initially, TESK1 was described as a Spry4-binding protein that co-localizes with Spry4 in endosomes [20]. Spry4 binds TESK1 via its C-terminal cysteine-rich domain and inhibits TESK1 activity resulting in decreased cell spreading [78]. TESK1 itself inhibits Spry2 activity by interfering with its subcellular localization so as to prevent serine dephosphorylation and also binding to Grb2 [77].

\section{Regulation of Spry protein stability and activity}

Spry protein stability and turnover are important determinants of how this family of negative feedback inhibitors affects endothelial cell homeostasis. While growth factor stimulation does induce Spry gene transcription [23, 24, 79], it also results in proteolytic degradation of Spry proteins $[45,46,80]$. This negative feedback loop system is thought to regulate Spry function temporally as a consequence of RTK stimulation [17, 18, 81]. Three recent studies have demonstrated that Spry stability is regulated in a complex manner [82-84]. As mentioned above, Spry proteins are phosphorylated on serine residues in unstimulated cells and are further phosphorylated on serine residues upon EGF and FGF stimulation [23, 82]. Phosphorylation of Spry2 at S112 and S121 is mediated by the MAPK-interacting kinase 1, Mnk1, which interferes with Spry2 tyrosine phosphorylation and thus stabilizes Spry2. Degradation of Spry2 is linked to phosphorylation of the conserved N-terminal tyrosine residue. A mutant version of Spry2 lacking all serine and tyrosine phosphorylation sites prevents Spry2 degradation, and a mutant version of Spry2 lacking the serine phosphorylation sites is no longer able to inhibit FGF-induced MAPK activation [82]. Together these data reveal that Spry stability and function require both tyrosine and serine phosphorylation.

Using a yeast two-hybrid screen, Nadeau and co-workers recently reported that Spry proteins interact with and are targets of the E3 ubiquitin ligase, "seven-in-absentia homolog 2" (SIAH2) [84]. Notably, SIAH2-mediated proteasomal degradation of Spry is independent of its tyrosine phosphorylation of the N-terminal tyrosine. These results uncover another mechanism for regulating Spry protein levels and function.

Finally, transforming growth factor-beta 1 (TGF- $\beta 1$ ) has been shown to regulate Spry2 at both the mRNA and protein levels [83]. TGF- $\beta 1$ plays a critical role in vessel maturation and in the initial stages of angiogenesis [85]. TGF- $\beta 1$-mediated degradation of endogenous Spry 2 protein is independent of MAPK activation, cannot be rescued by addition of EGF, and occurs in the lysosome. Furthermore, TGF- $\beta 1$ induces a transcriptional downregulation of Spry2 that is due to diminished mRNA production and requires de novo protein synthesis and histone deactylase activity. Lastly, Spry2's modulation of FGF and EGF signaling declined in cells pretreated with TGF- $\beta 1$, suggesting that TGF- $\beta$ signaling, possibly via the Smad proteins, can regulate Spry2 function by altering its mRNA and protein levels. In summary, Spry protein and mRNA turnover can be regulated by various signals, some of which are RTK-dependent while others are not (summarized in Fig. 2].

\section{Spry and nitric oxide}

Recently, it has been reported that Spry2 can assemble into large, spherical 24-mers with an iron-sulfur complex suggesting that they can function as redox sensors as these structures exist in oxidized, reduced, or nitrosylated forms
Fig. 2 Regulation of Spry2 stability. A diagram depicting the RTK-dependent and RTKindependent pathways that control Spry2 protein turnover. There are at least five signaling pathways that regulate Spry2 accumulation in the cell: transcriptional upregulation by the MAPK signaling pathway, serine phosphorylation by Mnk1, tyrosine phosphorylation by Src, ubiquitinylation by SIAH 2 , and TGF- $\beta 1$-mediated transcriptional downregulation and lysosomal degradation [23, 24, 79, 82-84]. pY55 denotes the $\mathrm{N}$-terminal tyrosine phosphorylation on Spry2

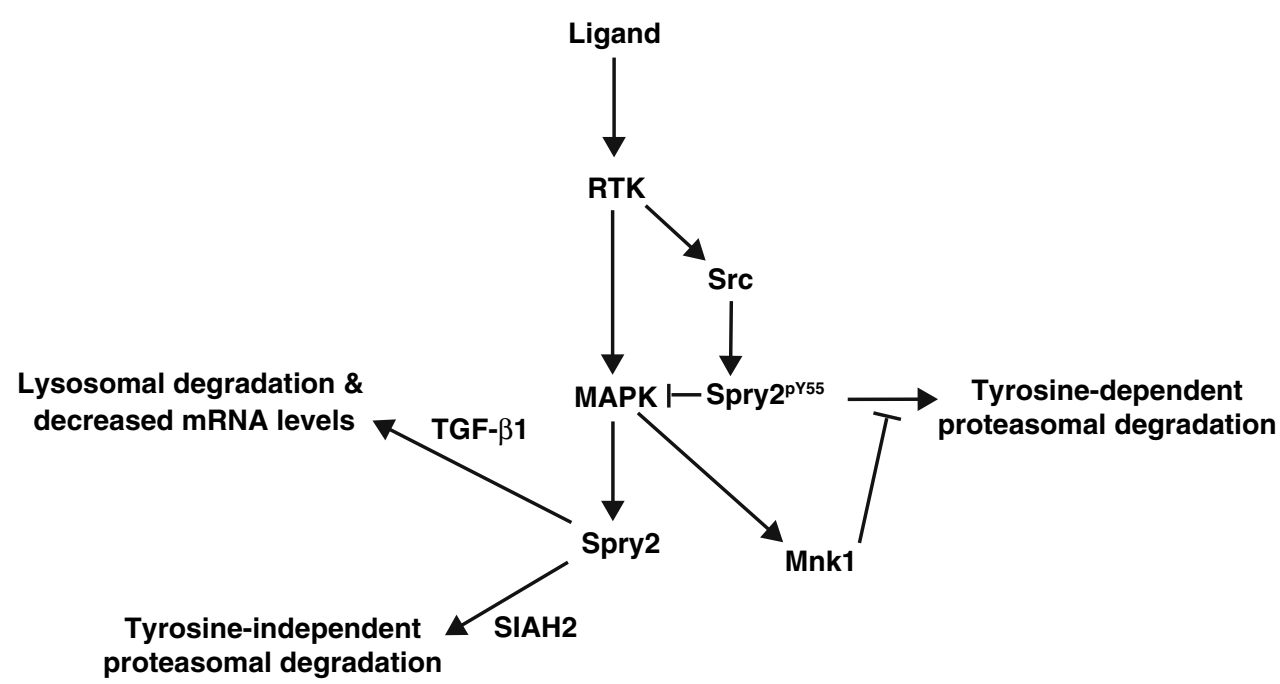


[86]. Notably, based on these observations it was proposed that these Spry2 complexes can hold an electrical charge and exhibit properties akin to an intracellular battery. Curiously, ectopic expression of nitric oxide synthase (NOS) in Drosophila mimics the dSpry mutation of increased tracheal branching [87]. In mammals, endothelial NOS (eNOS) is essential for endothelial cell proliferation and is required for vessel morphogenesis, branching, and stabilization (reviewed in [88]). Furthermore, the Spryinteracting protein, Caveolin-1, binds to and inhibits eNOS activity [89]. Thus, it is tempting to speculate that, in endothelial cells, Spry2 has additional functions that involve changes to its redox state and eNOS. To what extent, if any, these modifications affect Sprys' MAPK inhibitory activities has yet to be determined.

\section{Murine Spry knockout studies}

The generation of Spry null mice has yielded exciting new information about Spry function as well as confirming the central role of Sprys in modulating branching morphogenesis and growth factor signaling. Mice lacking Spry1 exhibit kidney and ureteric bud defects arising from improper branching morphogenesis [27, 90]. These malformations arise due to de-regulated signaling of GDNF by its cognate receptor c-Ret, which is essential for proper kidney development. Spry2 null mice have been generated independently in two laboratories. In one report, Spry2 null mice are viable, have a shortened life span, and exhibit hearing loss due to aberrant FGF8 signaling that results in malformation of the organ of Corti [91]. The other Spry2 knockout mouse line also exhibits a reduced lifespan and, in addition, a severe gastrointestinal phenotype characterized by enteric nerve dysplasia presumably due to GDNF hyperactivity [92].

More recently, two independent Spry4 null mouse lines have been reported $[93,94]$. In a first report, these mice were used to show that Spry2 and Spry4 control tooth development by antagonizing FGF signaling from different tissues; while Spry2 was expressed in the epithelium, Spry4 was found in the mesenchyme [93]. In a second report, Spry4 null mice showed reduced viability due to mandible defects, while the surviving mice exhibited growth retardation and polysyndactyly (i.e. fusion and duplication of forelimb digits) [94]. Also, Spry2/Spry4 double knockout mice were embryonic lethal with the majority of these embryos dying at E12.5 with apparent morphogenetic abnormalities of the head, lung and limbs, including cyclopia. Together, these data suggest that Spry2 and Spry4 have redundant activities during embryonic development. Although Spry overexpression markedly affects endothelial cell proliferation, tube formation, and angiogenesis in experimental models (see above), none of the Spry knockouts described thus far exhibits an overt vascular phenotype.

\section{Sprouty-related proteins, Spreds}

Spreds are related structurally and functionally to Spry proteins and have recently been reviewed [19]. Like Spry proteins, Spreds inhibit the MAPK pathway induced by various mitogens and share with Sprys the highly conserved cysteine-rich C-terminal SPR domain. Studies in Xenopus have highlighted the functional differences between Spry and Spred proteins. While in Xenopus Spry proteins inhibit PKC $\delta$ and calcium signaling, Spred proteins interfere with Ras-MAPK signaling [95]. In mammals, there seems to be more overlap in the signaling pathways that can be targeted by both Spry and Spreds, but Spred function tends to be more focused on MAPK inactivation [19]. A recent study examining the overlapping roles of Spred1 and Spred2 in double knockout mice demonstrates that Spreds are essential for development of the lymphatic system [96]. These mice are embryonic lethal at E12.5-E15.5 and display hemorrhages, edemas, and dilated lymphatic vessels, suggesting incomplete partitioning of lymphatic vessels from blood vessels. Expression of Spred1 and Spred2 is restricted to lymphatic endothelial cells, while Spry2 and Spry4 are found in both blood and lymphatic endothelial cells, suggesting that Spry function cannot replace Spred function in lymphatic endothelial cells. Apparently, Spred1 and Spred2 are able to repress the VEGF-C/VEGFR3-mediated signaling pathway. In contrast, Spry4 exerts only a very moderate inhibitory effect on this pathway. Curiously, both Spred proteins and Spry4 repress VEGF-A-induced MAPK activation via VEGFR2 [96]. In summary, while the Spred and Spry families share common features and activities, they have evolved to exert distinct and specific roles in modulating RTK signaling cascades.

\section{Summary and conclusions}

The critical contribution of Spry proteins in the control of signal transduction pathways in a variety of cell types, including endothelial cells, is well established. Nearly a decade has passed since they were first identified as antagonists of the FGF-induced signaling pathway. Ever since, their role in biology has expanded to encompass many more signaling pathways and biological processes, mostly involving an inhibitory function in RTK-induced MAPK signaling. While there are many open questions regarding Sprys' mechanisms of action in individual signaling pathways, it is now clear that Spry proteins are 
multi-functional and themselves highly regulated in their expression and function. Continuing studies will shed more light on the complexity of Spry function in controlling the multiple outputs of mitogen-induced cascades in physiological and pathological processes in general and in angiogenesis in particular. Novel insights about their molecular function may set the stage for the development of innovative therapeutic approaches to interfere with RTK-mediated pathological processes.

Acknowledgments The authors are grateful to Drs. Imke Albrecht, Anna Fantozzi, and Tibor Schomber for critically reading the manuscript. We apologize to all colleagues whose important work we could not cite due to space limitations. M.A.C. was initially supported by a post-doctoral fellowship from the International Agency for Research on Cancer (IARC-WHO). Research in the laboratory of the authors is supported by the Swiss National Science Foundation, Swiss Bridge Award, Krebsliga Beider Basel, Swiss Cancer League, EUFP6 Framework programmes LYMPHANGIOGENOMICS LSHGCT-2004-503573 and BRECOSM LSHC-CT-2004-503224, SNFNCCR Moleculary Oncology, and Novartis Pharma Inc.

\section{References}

1. Gschwind A, Fischer OM, Ullrich A (2004) The discovery of receptor tyrosine kinases: targets for cancer therapy. Nat Rev Cancer 4:361-370

2. Hanahan D, Weinberg RA (2000) The hallmarks of cancer. Cell 100:57-70

3. Carmeliet P (2005) Angiogenesis in life, disease and medicine. Nature 438:932-936

4. Hanahan D, Folkman J (1996) Patterns and emerging mechanisms of the angiogenic switch during tumorigenesis. Cell 86:353-364

5. Schlessinger J (2000) Cell signaling by receptor tyrosine kinases. Cell 103:211-225

6. Dor Y, Djonov V, Keshet E (2003) Making vascular networks in the adult: branching morphogenesis without a roadmap. Trends Cell Biol 13:131-136

7. Lu P, Sternlicht MD, Werb Z (2006) Comparative mechanisms of branching morphogenesis in diverse systems. J Mammary Gland Biol Neoplasia 11:213-228

8. Adams RH, Alitalo K (2007) Molecular regulation of angiogenesis and lymphangiogenesis. Nat Rev Mol Cell Biol 8:464-478

9. Ferrara N, Kerbel RS (2005) Angiogenesis as a therapeutic target. Nature 438:967-974

10. Dikic I, Giordano S (2003) Negative receptor signalling. Curr Opin Cell Biol 15:128-135

11. McKay MM, Morrison DK (2007) Integrating signals from RTKs to ERK/MAPK. Oncogene 26:3113-3121

12. Amit I, Citri A, Shay $T$ et al (2007) A module of negative feedback regulators defines growth factor signaling. Nat Genet 39:503-512

13. Hacohen N, Kramer S, Sutherland D et al (1998) sprouty encodes a novel antagonist of FGF signaling that patterns apical branching of the Drosophila airways. Cell 92:253-263

14. Bundschu K, Walter U, Schuh K (2006) The VASP-SpredSprouty domain puzzle. J Biol Chem 281:36477-36481

15. Cabrita MA, Christofori G (2003) Sprouty proteins: antagonists of endothelial cell signaling and more. Thromb Haemost 90:586-590

16. Guy GR, Wong ES, Yusoff P et al (2003) Sprouty: how does the branch manager work? J Cell Sci 16:3061-3068
17. Kim HJ, Bar-Sagi D (2004) Modulation of signalling by Sprouty: a developing story. Nat Rev Mol Cell Biol 5:441-450

18. Mason JM, Morrison DJ, Basson MA et al (2006) Sprouty proteins: multifaceted negative-feedback regulators of receptor tyrosine kinase signaling. Trends Cell Biol 16:45-54

19. Bundschu K, Walter U, Schuh K (2007) Getting a first clue about SPRED functions. Bioessays 29:897-907

20. Leeksma OC, Van Achterberg TA, Tsumura Y et al (2002) Human sprouty 4 , a new ras antagonist on $5 \mathrm{q} 31$, interacts with the dual specificity kinase TESK1. Eur J Biochem 269:2546-2556

21. Minowada G, Jarvis LA, Chi CL et al (1999) Vertebrate Sprouty genes are induced by FGF signaling and can cause chondrodysplasia when overexpressed. Development 126: 4465-4475

22. Gross I, Bassit B, Benezra M et al (2001) Mammalian sprouty proteins inhibit cell growth and differentiation by preventing ras activation. J Biol Chem 276:46460-46468

23. Impagnatiello MA, Weitzer S, Gannon G et al (2001) Mammalian sprouty-1 and -2 are membrane-anchored phosphoprotein inhibitors of growth factor signaling in endothelial cells. J Cell Biol 152:1087-1098

24. Sasaki A, Taketomi T, Wakioka $T$ et al (2001) Identification of a dominant negative mutant of Sprouty that potentiates fibroblast growth factor- but not epidermal growth factor-induced ERK activation. J Biol Chem 276:36804-36808

25. Lim J, Wong ES, Ong SH et al (2000) Sprouty proteins are targeted to membrane ruffles upon growth factor receptor tyrosine kinase activation. Identification of a novel translocation domain. J Biol Chem 275:32837-32845

26. Yigzaw Y, Cartin L, Pierre S et al (2001) The C terminus of sprouty is important for modulation of cellular migration and proliferation. J Biol Chem 276:22742-22747

27. Basson MA, Akbulut S, Watson-Johnson J et al (2005) Sprouty1 is a critical regulator of GDNF/RET-mediated kidney induction. Dev Cell 8:229-239

28. Gross I, Armant O, Benosman S et al (2007) Sprouty2 inhibits BDNF-induced signaling and modulates neuronal differentiation and survival. Cell Death Differ 14:1802-1812

29. Lee CC, Putnam AJ, Miranti CK et al (2004) Overexpression of sprouty 2 inhibits HGF/SF-mediated cell growth, invasion, migration, and cytokinesis. Oncogene 23:5193-5202

30. Choi H, Cho SY, Schwartz RH et al (2006) Dual effects of Sprouty 1 on TCR signaling depending on the differentiation state of the T cell. J Immunol 176:6034-6045

31. Glienke J, Schmitt AO, Pilarsky C et al (2000) Differential gene expression by endothelial cells in distinct angiogenic states. Eur J Biochem 267:2820-2830

32. Bell SE, Mavila A, Salazar R et al (2001) Differential gene expression during capillary morphogenesis in 3D collagen matrices: regulated expression of genes involved in basement membrane matrix assembly, cell cycle progression, cellular differentiation and G-protein signaling. J Cell Sci 114: 2755-2773

33. Jones N, Iljin K, Dumont DJ et al (2001) Tie receptors: new modulators of angiogenic and lymphangiogenic responses. Nat Rev Mol Cell Biol 2:257-267

34. Chi JT, Chang HY, Haraldsen G et al (2003) Endothelial cell diversity revealed by global expression profiling. Proc Natl Acad Sci U S A 100:10623-10628

35. Antoine M, Wirz W, Tag CG et al (2005) Expression pattern of fibroblast growth factors (FGFs), their receptors and antagonists in primary endothelial cells and vascular smooth muscle cells. Growth Factors 23:87-95

36. Paik JH, Kollipara R, Chu G et al (2007) FoxOs are lineagerestricted redundant tumor suppressors and regulate endothelial cell homeostasis. Cell 128:309-323 
37. Dejana E, Taddei A, Randi AM (2007) Foxs and Ets in the transcriptional regulation of endothelial cell differentiation and angiogenesis. Biochim Biophys Acta 1775:298-312

38. Ding W, Bellusci S, Shi W et al (2003) Functional analysis of the human Sprouty2 gene promoter. Gene 322:175-185

39. Lee SH, Schloss DJ, Jarvis L et al (2001) Inhibition of angiogenesis by a mouse sprouty protein. J Biol Chem 276:4128-4133

40. Christofori G (2003) Split personalities: the agonistic antagonist Sprouty. Nat Cell Biol 5:377-379

41. Cabrita MA, Jaggi F, Widjaja SP et al (2006) A functional interaction between sprouty proteins and caveolin-1. J Biol Chem 281:29201-2912

42. Lao DH, Chandramouli S, Yusoff P et al (2006) A Src homology 3-binding sequence on the $\mathrm{C}$ terminus of Sprouty2 is necessary for inhibition of the Ras/ERK pathway downstream of fibroblast growth factor receptor stimulation. J Biol Chem 281:2999330000

43. Ozaki K, Miyazaki S, Tanimura S et al (2005) Efficient suppression of FGF-2-induced ERK activation by the cooperative interaction among mammalian Sprouty isoforms. J Cell Sci 118:5861-5871

44. Egan JE, Hall AB, Yatsula BA et al (2002) The bimodal regulation of epidermal growth factor signaling by human Sprouty proteins. Proc Natl Acad Sci U S A 99:6041-6046

45. Fong CW, Leong HF, Wong ES et al (2003) Tyrosine phosphorylation of Sprouty2 enhances its interaction with c-Cbl and is crucial for its function. J Biol Chem 278:33456-33464

46. Rubin C, Litvak V, Medvedovsky H et al (2003) Sprouty finetunes EGF signaling through interlinked positive and negative feedback loops. Curr Biol 13:297-307

47. Schmelzle K, Kane S, Gridley S et al (2006) Temporal dynamics of tyrosine phosphorylation in insulin signaling. Diabetes 55:2171-2179

48. Mason JM, Morrison DJ, Bassit B et al (2004) Tyrosine phosphorylation of Sprouty proteins regulates their ability to inhibit growth factor signaling: a dual feedback loop. Mol Biol Cell 15:2176-2188

49. Hanafusa H, Torii S, Yasunaga T et al (2002) Sprouty1 and Sprouty 2 provide a control mechanism for the Ras/MAPK signalling pathway. Nat Cell Biol 4:850-858

50. Hanafusa H, Torii S, Yasunaga T et al (2004) Shp2, an SH2containing protein-tyrosine phosphatase, positively regulates receptor tyrosine kinase signaling by dephosphorylating and inactivating the inhibitor Sprouty. J Biol Chem 279:22992-22995

51. Jarvis LA, Toering SJ, Simon MA et al (2006) Sprouty proteins are in vivo targets of Corkscrew/SHP-2 tyrosine phosphatases. Development 133:1133-1142

52. Li X, Brunton VG, Burgar HR et al (2004) FRS2-dependent SRC activation is required for fibroblast growth factor receptorinduced phosphorylation of Sprouty and suppression of ERK activity. J Cell Sci 117:6007-6017

53. Presta M, Dell'Era P, Mitola S et al (2005) Fibroblast growth factor/fibroblast growth factor receptor system in angiogenesis. Cytokine Growth Factor Rev 16:159-178

54. Casci T, Vinos J, Freeman M (1999) Sprouty, an intracellular inhibitor of Ras signaling. Cell 96:655-665

55. Martinez N, Garcia-Dominguez CA, Domingo B et al (2007) Sprouty2 binds Grb2 at two different proline-rich regions, and the mechanism of ERK inhibition is independent of this interaction. Cell Signal 19:2277-2285

56. Rubin C, Zwang Y, Vaisman N et al (2005) Phosphorylation of carboxyl-terminal tyrosines modulates the specificity of Sprouty2 inhibition of different signaling pathways. J Biol Chem 280:9735-9744

57. Lao DH, Yusoff P, Chandramouli S et al (2007) Direct binding of PP2A to Sprouty2 and phosphorylation changes are a prerequisite for ERK inhibition downstream of fibroblast growth factor receptor stimulation. J Biol Chem 282:9117-9126

58. Amin DN, Hida K, Bielenberg DR et al (2006) Tumor endothelial cells express epidermal growth factor receptor (EGFR) but not ErbB3 and are responsive to EGF and to EGFR kinase inhibitors. Cancer Res 66:2173-2180

59. Sini P, Wyder L, Schnell C et al (2005) The antitumor and antiangiogenic activity of vascular endothelial growth factor receptor inhibition is potentiated by ErbB1 blockade. Clin Cancer Res 11:4521-4532

60. van Cruijsen H, Giaccone G, Hoekman K (2005) Epidermal growth factor receptor and angiogenesis: Opportunities for combined anticancer strategies. Int J Cancer 117:883-888

61. Wong ES, Fong CW, Lim J et al (2002) Sprouty2 attenuates epidermal growth factor receptor ubiquitylation and endocytosis, and consequently enhances Ras/ERK signalling. EMBO J 21:4796-4808

62. Wong ES, Lim J, Low BC et al (2001) Evidence for direct interaction between Sprouty and Cbl. J Biol Chem 276:58665875

63. Haglund K, Schmidt MH, Wong ES et al (2005) Sprouty2 acts at the $\mathrm{Cbl} / \mathrm{CIN} 85$ interface to inhibit epidermal growth factor receptor downregulation. EMBO Rep 6:635-641

64. Kim HJ, Taylor LJ, Bar-Sagi D (2007) Spatial regulation of EGFR signaling by Sprouty2. Curr Biol 17:455-461

65. Coultas L, Chawengsaksophak K, Rossant J (2005) Endothelial cells and VEGF in vascular development. Nature 438:937-945

66. Takahashi T, Yamaguchi S, Chida $\mathrm{K}$ et al (2001) A single autophosphorylation site on KDR/Flk-1 is essential for VEGF-Adependent activation of PLC-gamma and DNA synthesis in vascular endothelial cells. EMBO J 20:2768-2778

67. Sasaki A, Taketomi T, Kato R et al (2003) Mammalian Sprouty4 suppresses Ras-independent ERK activation by binding to Raf1. Nat Cell Biol 5:427-432

68. Parton RG, Simons K (2007) The multiple faces of caveolae. Nat Rev Mol Cell Biol 8:185-194

69. Bauer PM, Yu J, Chen Y et al (2005) Endothelial-specific expression of caveolin-1 impairs microvascular permeability and angiogenesis. Proc Natl Acad Sci U S A 102:204-209

70. Lin MI, Yu J, Murata T et al (2007) Caveolin-1-deficient mice have increased tumor microvascular permeability, angiogenesis, and growth. Cancer Res 67:2849-2856

71. Liu J, Wang XB, Park DS et al (2002) Caveolin-1 expression enhances endothelial capillary tubule formation. J Biol Chem 277:10661-10668

72. Woodman SE, Ashton AW, Schubert W et al (2003) Caveolin-1 knockout mice show an impaired angiogenic response to exogenous stimuli. Am J Pathol 162:2059-2068

73. Galbiati F, Volonte D, Engelman JA et al (1998) Targeted downregulation of caveolin-1 is sufficient to drive cell transformation and hyperactivate the p42/44 MAP kinase cascade. EMBO J 17:6633-6648

74. Kajita M, Ikeda W, Tamaru Y et al (2007) Regulation of plateletderived growth factor-induced Ras signaling by poliovirus receptor Necl-5 and negative growth regulator Sprouty2. Genes Cells 12:345-357

75. Sakisaka T, Ikeda W, Ogita $\mathrm{H}$ et al (2007) The roles of nectins in cell adhesions: cooperation with other cell adhesion molecules and growth factor receptors. Curr Opin Cell Biol 19:593-602

76. Couderc T, Barzu T, Horaud F et al (1990) Poliovirus permissivity and specific receptor expression on human endothelial cells. Virology 174:95-102

77. Chandramouli S, Yu CY, Yusoff P et al (2008) Tesk1 interacts with sprouty2 to abrogate its inhibition of ERK phosphorylation downstream of receptor tyrosine kinase signaling. J Biol Chem 283:1679-1691 
78. Tsumura Y, Toshima J, Leeksma OC et al (2005) Sprouty-4 negatively regulates cell spreading by inhibiting the kinase activity of testicular protein kinase. Biochem J 387:627-637

79. Ozaki K, Kadomoto R, Asato K et al (2001) ERK pathway positively regulates the expression of Sprouty genes. Biochem Biophys Res Commun 285:1084-1088

80. Hall AB, Jura N, DaSilva J et al (2003) hSpry2 is targeted to the ubiquitin-dependent proteasome pathway by $\mathrm{c}$-Cbl. Curr Biol 13:308-314

81. Rubin C, Gur G, Yarden Y (2005) Negative regulation of receptor tyrosine kinases: unexpected links to $\mathrm{c}-\mathrm{Cbl}$ and receptor ubiquitylation. Cell Res 15:66-71

82. DaSilva J, Xu L, Kim HJ et al (2006) Regulation of sprouty stability by Mnk1-dependent phosphorylation. Mol Cell Biol 26:1898-1907

83. Ding W, Shi W, Bellusci S et al (2007) Sprouty2 downregulation plays a pivotal role in mediating crosstalk between TGF-beta1 signaling and EGF as well as FGF receptor tyrosine kinase-ERK pathways in mesenchymal cells. J Cell Physiol 212:796-806

84. Nadeau RJ, Toher JL, Yang X et al (2007) Regulation of Sprouty2 stability by mammalian Seven-in-Absentia homolog 2. J Cell Biochem 100:151-160

85. Jain RK (2003) Molecular regulation of vessel maturation. Nat Med 9:685-693

86. Wu X, Alexander PB, He Y et al (2005) Mammalian sprouty proteins assemble into large monodisperse particles having the properties of intracellular nanobatteries. Proc Natl Acad Sci U S A 102:14058-14062

87. Wingrove JA, O'Farrell PH (1999) Nitric oxide contributes to behavioral, cellular, and developmental responses to low oxygen in Drosophila. Cell 98:105-114
88. Ying L, Hofseth LJ (2007) An emerging role for endothelial nitric oxide synthase in chronic inflammation and cancer. Cancer Res 67:1407-1410

89. Minshall RD, Sessa WC, Stan RV et al (2003) Caveolin regulation of endothelial function. Am J Physiol Lung Cell Mol Physiol 285:L1179-L1183

90. Basson MA, Watson-Johnson J, Shakya R et al (2006) Branching morphogenesis of the ureteric epithelium during kidney development is coordinated by the opposing functions of GDNF and Sprouty1. Dev Biol 299:466-477

91. Shim K, Minowada G, Coling DE et al (2005) Sprouty2, a mouse deafness gene, regulates cell fate decisions in the auditory sensory epithelium by antagonizing FGF signaling. Dev Cell 8:553564

92. Taketomi T, Yoshiga D, Taniguchi K et al (2005) Loss of mammalian Sprouty2 leads to enteric neuronal hyperplasia and esophageal achalasia. Nat Neurosci 8:855-857

93. Klein OD, Minowada G, Peterkova R et al (2006) Sprouty genes control diastema tooth development via bidirectional antagonism of epithelial-mesenchymal FGF signaling. Dev Cell 11:181-190

94. Taniguchi K, Ayada T, Ichiyama K et al (2007) Sprouty2 and Sprouty4 are essential for embryonic morphogenesis and regulation of FGF signaling. Biochem Biophys Res Commun 352:896-902

95. Sivak JM, Petersen LF, Amaya E (2005) FGF signal interpretation is directed by Sprouty and Spred proteins during mesoderm formation. Dev Cell 8:689-701

96. Taniguchi K, Kohno R, Ayada T et al (2007) Spreds are essential for embryonic lymphangiogenesis by regulating vascular endothelial growth factor receptor 3 signaling. Mol Cell Biol 27:45414550 